\title{
Microporous Binder for Silicon Based Lithium-Ion Battery Anode with Exceptional Rate Capability and Improved Cyclic Performance
}

Liujia Ma ${ }^{1,2}$, Jianqiang Meng ${ }^{1 *}$, Ying Pan ${ }^{1}$, Ya-Jun Cheng ${ }^{2}{ }^{3 *}$, Qing $\mathrm{Ji}^{2}, 4$, Xiuxia $\mathrm{Zuo}^{2 *}$, Xiaoyan Wang ${ }^{2}$, Jin Zhu' ${ }^{2}$, Yonggao $\mathrm{Xia}^{2}$, $^{*}$

1. State Key Laboratory of Separation Membranes and Membrane Processes, Tianjin Polytechnic University, Tianjin 300387, People’s Republic of China

2. Ningbo Institute of Materials Technology and Engineering, Chinese Academy of Sciences, 1219 Zhongguan West Road, Zhenhai District, Ningbo, Zhejiang Province 315201, People's Republic of China

3. Department of Materials, University of Oxford, Parks Rd, OX1 3PH, Oxford, United Kingdom

4. The University of Nottingham Ningbo China, 199 Taikang East Road, Ningbo, Zhejiang Province 315100, People's Republic of China

5. Center of Materials Science and Optoelectronics Engineering, University of Chinese Academy of Sciences, 19A Yuquan Rd, Shijingshan District, Beijing 100049, People's Republic of China

Email_address:_jianqiang.meng@hotmail.com,_chengyj@nimte.ac.cn, 
zuoxiuxia@nimte.ac.cn, xiayg@nimte.ac.cn

Number of pages: 9

Number of figures: 5

Number of schemes: 0

Number of tables: 0 
Materials: 5,5',6,6'-Tetrahydroxy-3,3,3',3'-tetramethyl-1,1'-spirobisindane (TTSBI, 99\%) was purchased from Alfa Aesar (Shanghai, China). 2,3,5,6-Tetrafluoroterephthalonitrile (TFTPN, 98\%) and anhydrous potassium carbonate $\left(\mathrm{K}_{2} \mathrm{CO}_{3}, \mathrm{CR}\right)$ were purchased from Sigma-Aldrich (USA). Sodium hydroxide $(\mathrm{NaOH}, \mathrm{AR})$ and hydrochloric acid standard solution $(0.01 \mathrm{~mol}, \mathrm{AR})$ were bought from Kermel Chemical Reagent Co. Ltd. (Tianjin, China). TTSBI and TFTPN need further purification before use. The pristine monomer of TTSBI was dissolved in methanol for recrystallization. The obtained white powder was collected by vacuum filtration and placed in vacuum oven at $60{ }^{\circ} \mathrm{C}$ for $48 \mathrm{~h}$ until no mass lass was observed. TFTPN was purified by vacuum sublimation at $145^{\circ} \mathrm{C}$ in oil bath. Both TTSBI and TFTPN were kept in a desiccator for storage.

Sample preparetion:The synthesis procedure of PIM-1 was conducted as follows ${ }^{1}$. A mixture of purified TTSBI (3.404 g), TFTPN (2.001 g) and anhydrous $\mathrm{K}_{2} \mathrm{CO}_{3}(2.820$ g) with a molar ratio of 1: 1: 2.04 was added to $70 \mathrm{~mL}$ of dry DMF. The solution was stirred at $65{ }^{\circ} \mathrm{C}$ for $48 \mathrm{~h}$ under $\mathrm{N}_{2}$ atmosphere. Then the solution was kept still until a yellow precipitate was observed. The precipitate was collected by vacuum filtration and rinsed with methyl alcohol for several times to obtain PIM-1. The hydrolysis procedure of the as-prepared PIM-1 was performed by refluxing the PIM-1 powder in a $10 \mathrm{wt} \% \mathrm{NaOH}$ solution $\left(\mathrm{H}_{2} \mathrm{O} / \mathrm{ethanol}=1: 1(\mathrm{v} / \mathrm{v})\right)$ at $120^{\circ} \mathrm{C}$ for $5 \mathrm{~h}$ with stirring ${ }^{2}$. Upon hydrolysis, the colour of the sample appearance changed from bright-yellow to dark-yellow. Then the solution of the hydrolyzed sample (PIM-COONa) was titrated to $\mathrm{pH}$ neutral and the powder was collected and dried at $120{ }^{\circ} \mathrm{C}$. The results are 
depicted in the figure below (Figure S1 and S2). 


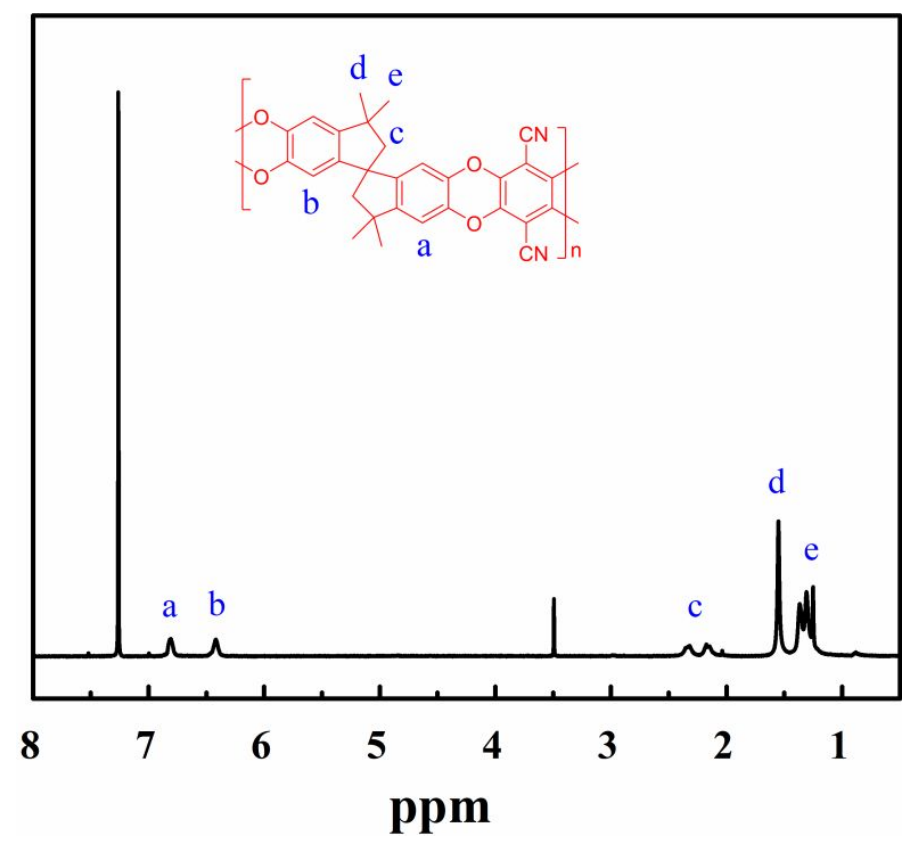

Figure S1. The ${ }^{1} \mathrm{H}$ NMR spectra of PIM-1.

${ }^{1} \mathrm{H}$ NMR analysis of the PIM-1 is shown in Figure S1. The peaks at $6.8 \mathrm{ppm}$ (a) and $6.4 \mathrm{ppm}(\mathrm{b})$ are ascribed to protons in the benzene ring of the PIM-1 molecular chain. The peaks of the $2.15 \mathrm{ppm}-2.50 \mathrm{ppm}$ (c) correspond to $-\mathrm{CH}_{2}$ - in the pentabasic cyclic structure, while the peaks of $1.50 \mathrm{ppm}$ (d) and $1.20 \mathrm{ppm}$ (e) correspond to two adjacent $-\mathrm{CH}_{3}-$. The peaks in the $2.80 \mathrm{ppm}-2.90 \mathrm{ppm}$ region are ascribed to the DMF residuals. 




Figure S2. FT-IR spectra of PIM-1 and PIM-COOH

The absorption bands in the region of $1124 \mathrm{~cm}^{-1}-878 \mathrm{~cm}^{-1}$ are attributed to the stretching vibration of dioxane produced by condensation polymerization of TTSBI and TFTPN. The region of $2970 \mathrm{~cm}^{-1}-2840 \mathrm{~cm}^{-1}$ is corresponding to stretching of $-\mathrm{CH}_{3}$ in the PIM-1 chain. The absorption band at $2245 \mathrm{~cm}^{-1}$ is ascribed to the $-\mathrm{CN}$ stretching vibration. After hydrolysis, the peak corresponding to the $-\mathrm{CN}$ stretching vibration disappears and a broad peak locates between $3000 \mathrm{~cm}^{-1}-3500 \mathrm{~cm}^{-1}$ is ascribed to the $-\mathrm{COOH}$ in the PIM-COOH polymer chain. 
(a)

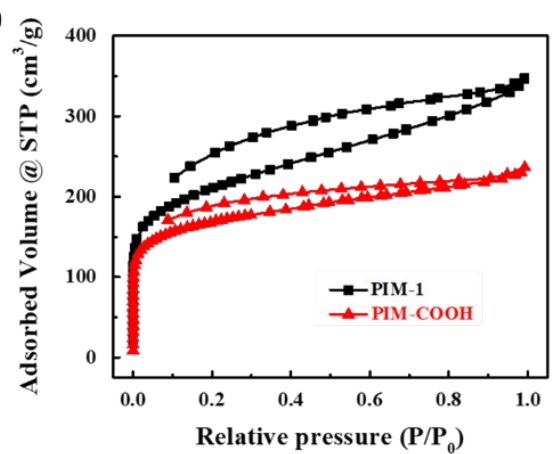

(b)

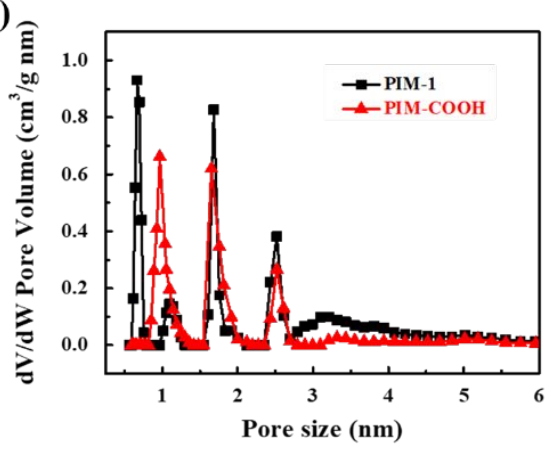

Figure S3. $\mathrm{N}_{2}$ adsorption-desorption isotherms (a) and pore size distribution (b) of PIM-1 and PIM-COOH samples.

$\mathrm{N}_{2}$ adsorption-desorption isotherms and pore size distribution of the PIM-1 and PIM-COOH samples are shown in Figure S3a and b. The original PIM-1 sample has a high surface area of $760 \mathrm{~m}^{2} \cdot \mathrm{g}^{-1}$ due to its intrinsic microporous structure. After hydrolysis, the surface area of PIM-COOH decreases to $547 \mathrm{~m}^{2} \cdot \mathrm{g}^{-1}$. The electrostatic interaction and hydrogen bond induced by hydrolysis increase the intermolecular interaction, leading to slight reduction of surface area and free volume $e^{3,4}$.
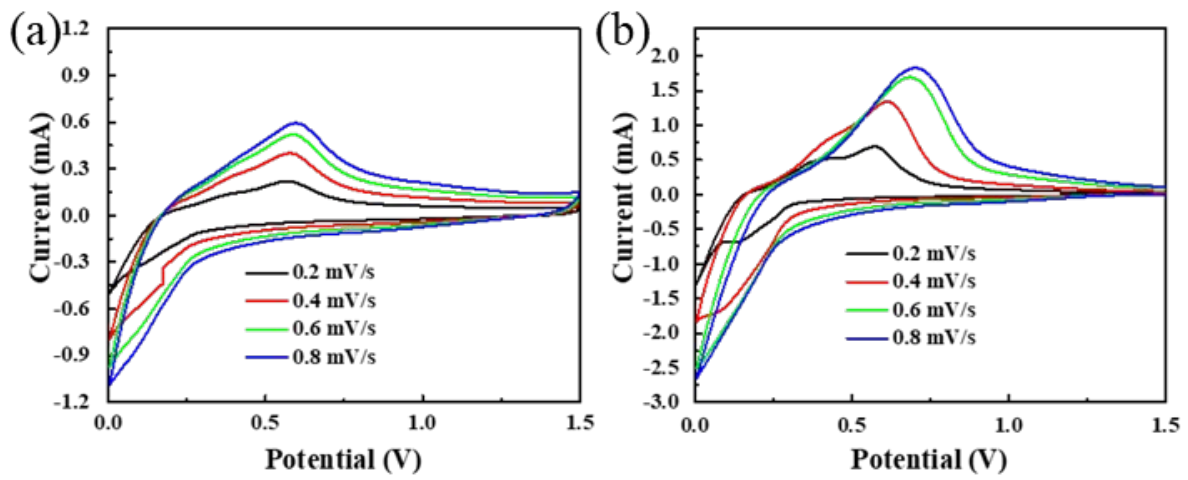

Figure S4. CV curves of (a) PIM-COOH/Si and (b) SA/Si electrodes at different scan rates. 




Figure S5. Linear fits for the anodic peak current versus square root of scan rate of $\mathrm{PIM}-\mathrm{COOH} / \mathrm{Si}$ and $\mathrm{SA} / \mathrm{Si}$ electrodes.

The Linear fits for the anodic peak current versus square root of scan rate were obtained from the result in Figure S5. 


\section{Reference}

(1) Pan, Y.; Zhai, X.; Yin, J.; Zhang, T.; Ma, L.; Zhou, Y.; Zhang, Y.; Meng, J. Hierarchical Porous and Zinc-Ion-Crosslinked PIM-1 Nanocomposite as a $\mathrm{CO}_{2}$ Cycloaddition Catalyst with High Efficiency. ChemSusChem, 2019, 12, 2231-2239.

(2) Du, N.; Robertson, G.; Song, J.; Pinnau, I.; Guiver, M. High-Performance Carboxylated Polymers of Intrinsic Microporosity (PIMs) with Tunable Gas Transport Properties. Macromolecules, 2009, 42, (16), 584-588.

(3) Weber, J.; Du, N.; Guiver, M. D. Influence of Intermolecular Interactions on the Observable Porosity in Intrinsically Microporous Polymers. Macromolecules, 2011, 44, (7), 1763-1767.

(4) Satilmis, B.; Budd, P. M. Base-Catalysed Hydrolysis of PIM-1: Amide versus Carboxylate Formation. RSC Adv., 2014, 4, (94), 52189-52198. 